\title{
Prácticas docentes y educación multigrado en escuelas indígenas del Estado de Sonora, México*
}

\author{
José Ángel Vera Noriega ${ }^{1}$ \\ orcid.org/0000-0003-2764-4431 \\ Carlos Enrique Peña Meléndez ${ }^{2}$ \\ orcid.org/0000-0001-6605-1781 \\ Centro de Investigación en Alimentación y Desarrollo A.C., México \\ DOI: http://dx.doi.org/10.17081/eduhum.18.31.1376
}

Recibido: 4 de marzo de 2016

Aceptado: 22 de julio de 2016

\section{Teaching work and multigrade education in native indian schools at the State of Sonora, Mexico}

Palabras clave:

Escuela multigrado, Comunidad indígena,

Prácticas docentes,

Condición laboral.

\begin{abstract}
Resumen
Se plantea como objetivo de investigación generar conocimientos que permitan comprender las relaciones existentes entre el perfil docente y el rezago/deserción educativa, a fin de proponer ideas que mejoren las condiciones de trabajo de los maestros que laboran en contextos sociales de marginación social dentro de las zonas rurales indígenas. Para llevarlo a cabo y hacer la observación de las distintas prácticas docentes y el contexto educativo en que estos profesores laboran, se seleccionaron tres escuelas que obtuvieron los puntajes más bajos en español y matemáticas en la prueba Enlace si se comparan con las demás escuelas de la zona. Se identificó que los docentes de estas tres escuelas multigrado intentan tanto mantener en sus enseñanzas la tradición cultural de la tribu como responder a las exigencias curriculares para cada grado, además de cumplir con una doble labor: docente y directivo dentro de la misma institución.
\end{abstract}

Keywords:

Multigrade school, Indian community, Teacher's training, Working conditions.

\begin{abstract}
The main object of this research work is focused on the creation of enough knowledgements that allow the understanding of the relationship between teacher's profile and school drop-outs/student desertions. All of these, with the final idea of making proposals to improve teacher's working conditions, particularly those that are working in contextual marginal outcast areas within Indian rural zones. In order to carry out and make the different teacher's class observations under the educative context where they work, three schools were carefully selected that had obtained the lowest scores in Spanish and Maths in the ENLACE TEST compared to other schools in the zone. It was observed that the teachers of these multigrade schools maintained their traditional Indian tribe culture at teaching in response to curricular demands for each grade, besides the fact that they also complied with the double function: teacher and executive within the same institution.
\end{abstract}

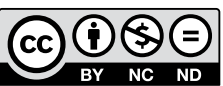

Referencia de este artículo (APA): Vera, J. A. \& Peña, C. E. (2016). Prácticas docentes y educación multigrado en escuelas indígenas del Estado de Sonora, México. Revista Educación y Humanismo, 18(31), 225-240. http://dx.doi.org/10.17081/eduhum.18.31.1376

\footnotetext{
* Este artículo es resultado del proyecto Perfil y condiciones de trabajo de profesores que atienden escuelas primarias multigrado con rezago educativo en San Luis Potosí, Chiapas y Sonora, que contó con la colaboración de tres instituciones distintas: la Universidad Autónoma de San Luis Potosí, la Universidad Autónoma de Chiapas y el Centro de Investigación en Alimentación y Desarrollo A.C. Centro Proyecto del Fondo Sectorial de Investigación para la Educación SEP/SEB-Conacyt, 2009. Número de proyecto: 000000000144944.

1. Doctor en Psicología, Licenciado en Psicología. Investigador titular del Centro de Investigación en Alimentación y Desarrollo A.C. Email: avera@ciad.mx

2. Magíster en Desarrollo Regional (c), Licenciado en Psicología. Asistente de Investigación del Centro de Investigación en Alimentación y Desarrollo A.C. Email: cpm_33@hotmail.com
} 


\section{Introducción}

La escuela rural multigrado y particularmente la pluricultural requiere pensarse como un universo conceptual independiente, pero cuidando de no confinar en él a una población mayoritariamente indígena, que se expresa mediante un conjunto de mitos, ritos y tradiciones propios del mexicanismo, pues así busca que en sus alumnos la institución educativa vaya estructurando más, mejores y diferentes manifestaciones de identidad nacional. Es importante partir de un acuerdo sobre lo que esperamos de una escuela de este tipo: con respecto al desarrollo de habilidades lógicas y de la lectoescritura, que procuren contribuir con el mantenimiento de sus procesos de identificación social y personal. También se esperaría una actitud reflexiva y crítica que frente a su situación indígena y rural sirva para reproducir los procesos sociales de comunicación, educación, salud, religión, crianza, relaciones de pareja y otros (Vera, Ramírez \& Laborín, 2010).

¿Qué necesita aprender un niño indígena en un grupo multigrado con su profesor en el proceso de enseñanza-aprendizaje? Primero, todo sobre la historia de sus antepasados, sobre sus trayectorias sociales y tradición oral, además de las bases comunitarias y colectivas que hicieron posible su subsistencia. Segundo, necesita aprender a reflexionar sobre sus proyectos, sus producciones narrativas y las bases comunitarias y colectivas que hacen posible su sobrevivencia. Tercero, requiere ser capaz de planear un proyecto familiar o colectivo y ser capaz de escribirlo y narrarlo. Creemos que esto podría ser el objetivo de la escuela multigrado, la integración e identificación del niño con su comunidad y la posibilidad de una reflexión sobre su pasado, su presente y su futuro (Peña, Pérez \& Vera, 2008).

No obstante, las técnicas educativas, contenidos y en general el modelo curricular de dos ciclos de la escuela de niños multigrado, aun cuando está moldeado sobre condiciones ejemplares de la cotidianidad del niño, el modelo formativo en habilidades académicas básicas es muy parecido al de la escuela urbana de clase media (Juárez, 2009). En este ambiente todas las aspiraciones sociales y de los maestros van dirigidas a la lectoescritura y matemáticas, leer, escribir, sumar y restar. Los maestros se enfocan a estas competencias básicas, y cuando encuentran niños que ya las dominan, buscan su ayuda para soportar los procesos de los más pequeños. Los esfuerzos iniciales por la enseñanza de tópicos curriculares adscritos a cuarto, quinto $\mathrm{y}$ sexto son poco estimulantes, pues los padres tienen pocas habilidades para apoyar a los hijos siguiendo el esfuerzo de los maestros. Además, el ciclo de enseñanza es corto, el número de horas clase es mínimo y la insistencia por la urgencia de los pagos salariales mantiene al profesor en una condición de poca motivación, frente a un esfuerzo máximo con pocas habilidades en sus alumnos.

Entretanto las estrategias didácticas, motivacionales de planeación y evaluación que les propone el programa diseñado por la Secretaría de Educación Pública (SEP) no ofrecen una alter- 
nativa coherente, relevante y pertinente para enfrentar esta realidad en el aula (Instituto Nacional para la Evaluación de la Educación [INEE], 2006).

El reconocimiento de este contexto es importante para el diseño y planificación de estrategias de cambio de conocimientos, actitudes y conductas en la medida que constituye un problema de investigación que debe documentarse desde el ámbito de la política educativa hasta el trabajo en aula.

En estas comunidades rurales e indígenas, la abundancia de problemas relacionados con el rezago educativo, la disposición al riesgo y la carencia de motivación al logro están relacionados con el nivel educativo alcanzado por la madre y el número de hijos; entre mayor sea la cantidad de hijos, mayor será la sobrecarga de trabajo en el hogar y, por lo tanto, habrá menos tiempo disponible para dedicar al cuidado y educación del niño, así como la generalización de prácticas y creencias inadecuadas sobre su desarrollo (Launtrey, 1985).

Otro factor importante que influye en el rendimiento académico del niño refiere a las características de la escuela y el aula, en tanto definen su carácter social; por ejemplo: las actitudes de los maestros, los valores de la escuela, el clima de la escuela y el aula son fuentes significativas de variación en el rendimiento de los estudiantes. Según Schmelkes (1996), en 1995 el 8 \% de los niños mexicanos entre 6 y 14 años (edad de la educación primaria) no estaba en la escuela.

\section{Situación de la educación rural indígena}

En México, el Instituto Nacional de Estadística y Geografía [INEGI] define ruralidad a partir del número de habitantes que residen en una localidad (menor a 2.500 habitantes). Del total de 187.938 localidades habitadas según el Conteo de Población y Vivienda del 2005, solamente 550 son consideradas urbanas; sin embargo, a pesar de este alto número de localidades rurales, el número de habitantes en ellas ha disminuido en más de un $30 \%$ de 1950 a la actualidad (Sánchez, 2011; INEGI, 2011). Dentro de estas localidades rurales la distribución de la población según el grado de presencia indígena es: 74,3 \% es escasa, $14,7 \%$ son indígenas, $7,3 \%$ moderada y $3,8 \%$ predominantemente indígenas (INEGI, 2010).

Esta relación entre poblaciones rurales e indígenas se establece a partir de la vulnerabilidad social a la que están sujetas, sus desventajas sociales, económicas y políticas en comparación con las ciudades. Dentro de estos contextos similares (indígena y rural) "ser indígena" refiere a los procesos de construcción de identidad, los cuales son dinámicos y se asocian a aspectos económicos, políticos, históricos y culturales. Sin embargo, aún existe una discusión institucional sobre los criterios que definen a una población como indígena (Robles, Hernández \& Godínez, 2009).

En una población rural e indígena se presentan condiciones socioeconómicas muy diferentes a las poblaciones urbanas, estos contrastes 
están presentes en el capital cultural de las familias que las integran. En una zona rural, las condiciones sociodemográficas desfavorables en las que habitan los pobladores no les permiten tener la misma calidad de educación a la que tienen acceso los ciudadanos en las grandes urbes (Sánchez, 2011; Peña, 2004).

En este tipo de comunidades los problemas de rezago educativo, la disposición al riesgo y la carencia de motivación al logro están relacionados con el nivel educativo alcanzado por la madre y con el número de hijos. De acuerdo con el INEGI (2010) el 35,5\% de la población rural se encuentra en rezago educativo, como consecuencia, también, de la falta de infraestructura educativa y de la dispersión que existe entre estas comunidades lo que dificulta el acceso y la provisión de servicios educativos públicos. Diversas investigaciones en el área de la educación rural señalan que los factores desfavorables del contexto rural pueden estar asociados a bajos resultados escolares de la población (Guerra, 2005; Comisión Nacional para el Desarrollo de los Pueblos Indígenas [CDI], 2014).

Rodríguez (2006) presenta los siguientes datos: en la zona rural existen menos maestros con maestría, pero todos ellos estudiaron cuatro años en una escuela para obtener el grado de profesor. Los profesores de la zona rural o serrana no recibieron cursos de capacitación o actualización sobre el quehacer en el aula, por lo cual existe un desfase temporal y de contenidos; adicionalmente, como estos docentes tienen rotaciones más frecuentes que en la zona capitalina y frontera, no pueden completar un ciclo de aculturación que les permita conocer las necesidades del estudiante pobre y marginal de la localidad (Vera \& Domínguez, 2005). La calidad requiere de igualdad para que los niveles educativos mejoren, bien sea reduciendo el rezago, o bien, mejorando el desempeño de aprendizaje, para lo cual es necesario que las zonas rurales e indígenas se igualen en condiciones de insumos y procesos con las mejores de la zona urbana (Búrquez, Domínguez \& Vera, 2005).

Considerar las características del personal docente que atiende las aulas de niños multigrado y los servicios de educación indígena resulta relevante en la medida en que aquel, el docente, es un elemento fundamental para el desarrollo del proceso educativo en todas sus dimensiones de intervención: los procesos de enseñanza y de aprendizaje, la gestión escolar, la promoción de la participación social y la traducción, adaptación y operación de los fines y propósitos de la educación básica delimitados en el Programa Nacional de Educación y, de manera más específica, en los Planes y Programas de Estudio (López \& Viveros, 2002).

Se plantea como objetivo de investigación, generar conocimientos que permitan comprender las relaciones existentes entre el perfil docente y el rezago/deserción educativa, a fin de proponer ideas que mejoren las condiciones de trabajo de los maestros que laboran en contextos sociales de marginación social dentro de las zonas rurales indígenas. 


\section{Método}

El estudio se ha llevado a cabo utilizando la metodología etnográfica de investigación, ya que dadas sus características se consideró que podía ser la más acertada en este caso (Atkinson \& Hammersley, 1994), en donde se busca comprender en profundidad los fenómenos educativos que se modifican en la escuela multigrado.

\section{Población}

Contexto socioeconómico y geográfico de las comunidades seleccionadas (ver en Anexos Tabla 1).

Para el estudio que se plantea se seleccionaron tres escuelas multigrado dentro de la modalidad indígena ubicadas dentro de las gubernaturas tradicionales yaquis de Belem y Pótam. Las tres comunidades pertenecen al municipio de Guaymas, pero se encuentran en la frontera con el municipio de Cajeme, lo cual desata un sistema de gestión confuso.

Actualmente existe un conflicto de dualidad en las autoridades de la tribu; por un lado, se encuentra la resistencia al abandono de la estructura tradicional, sus lineamientos de autoridades y sus formas consensos, y, por el otro lado, un proceso de diversificación de intereses donde prevalece tanto la individualización de beneficios y decisiones (frente a lo grupal) como la aceptación de nuevas formas de corporación impuestas desde el exterior.

Esta confrontación de facciones constituye la lucha por el poder económico y político del pueblo yaqui, que puede notarse en la oposición a las normas y mecanismos de la estructura de organización tradicional.

Las comunidades no cuentan con drenaje; carecen de calles pavimentadas, excepto la carretera lascruza. En 7 de cada 10 casas se observa que existe una fosa séptica, pero muy pocas drenan el agua de lavado de ropa y de cocina hacia esta, sino más bien a flor de tierra, que se escurre en la vía pública provocando malos olores y problemas de sanidad. En su mayoría, las viviendas están construidas con carrizo y una mezcla de lodo a base de tierra, arena y estiércol, techo de lámina negra con estructura de horcones en su mayoría de mezquite y techo de lámina negra, cubierto de tierra. El orden interior de las habitaciones es muy precario porque apenas tienen lo básico en vestuario y electrónicos, que se distribuyen en espacios saturados de bolsas de poliuretano o en tapancos improvisados.

La comunidad de Rahum, Guaymas, registra un alto grado de marginación: tiene una población total de 272 habitantes, que viven en 59 viviendas particulares de las cuales $3,38 \%$ de las unidades no cuenta con escusado ni energía eléctrica; $8,62 \%$ no tiene el servicio de agua entubada; $74,57 \%$ de las casas conserva piso de tierra y $54,23 \%$ carece de refrigerador para mantener en buen estado sus alimentos.

La comunidad de Huirivis, Guaymas, presenta el más alto grado de marginación de las tres 
comunidades en observación; contabiliza un total de 342 habitantes, y de las 71 viviendas habitadas está sin retrete el 11,26\%, 2,81\% no recibe servicio de agua entubada y el $10 \%$ tampoco tiene energía eléctrica; $60,56 \%$ muestra piso de tierra y $49,29 \%$ no cuenta con refrigerador.

A su vez, Pitahaya, Guaymas, con un alto grado de marginación tiene una población total de 285 habitantes y 60 viviendas particulares; se sitúa como la comunidad con las condiciones más deficientes de infraestructura en comparación con las otras dos ya nombradas. Apenas el 28,33 $\%$ del total de las casas solo tiene letrina, $15 \%$ no recibe servicio de energía eléctrica y menos aún las instalaciones de la escuela primaria; $3,33 \%$ no cuenta con servicio de agua entubada; el piso del 68,33\% de las viviendas es de tierra y el 71,66\% de las casas habitadas no dispone de refrigerador para conservar los alimentos.

\section{Población escolar}

Las tres escuelas donde se trabajó son las siguientes: (ver Anexos Tabla 2).

a) Escuela A (Primaria General Ignacio Mori, Pitahaya).

b) Escuela B (Primaria Gabriel Zapajiza, Huirivis).

c) Escuela C (Primaria José María Matus, Rahum).

Considerando que el tercer grado representa el final de la alfabetización desde el punto de vista de los programas de la Reforma Integral de la Educación Básica (RIEB), y el sexto un pun- to de corte para la comprensión de lectura, estas escuelas han mantenido en Español para tercero y sexto grado entre 2010 y 2012 un nivel insuficiente y un nivel elemental en tercero (ver en Anexos Tabla 3).

En Matemáticas, para sexto grado, durante el 2011 se mantuvieron los mejores niveles de logro en el nivel elemental y bueno (ver en Anexos Tabla 4).

\section{Levantamiento de la información}

Después de decidir sobre las escuelas, se realizaron seis visitas de una semana cada una (ver en Anexos Tabla 5).

Para el levantamiento de la información de las actividades didácticas fue necesario dividir las observaciones en dos tipos: una enfocada a la observación de la clase relacionada con el contenido curricular y la otra centrada en las actividades pedagógicas planeadas por el docente.

Esto se llevó a cabo de la siguiente manera: durante las tres semanas de trabajo de campo se realizaron dos sesiones de observación en distintas clases por cada salón en cada una de las tres primarias, cada una enfocaba lo requerido ya sea observación de clase o actividades pedagógicas.

Diez de las sesiones de observación didáctica se realizaron entre 8 y 10 a.m. con una duración aproximada que iba desde 60 hasta 80 minutos cada una; las dos sesiones de observación restantes se hicieron durante las 10 de la mañana y las 12 de la tarde. 
Sin excepción alguna, las entrevistas realizadas a maestros, directores, estudiantes y padres de familia se hicieron con grabadora digital durante todo el tiempo que duró el trabajo, para así poder almacenar cada grabación en computador en formato MP3. Al terminar con la totalidad de las entrevistas se procedió a su transcripción en documentos de Word.

Con el objetivo de categorizar los contenidos de las entrevistas se llevó a cabo un análisis de Fortalezas, Oportunidades, Debilidades y Amenazas (FODA) de las condiciones pedagógicas y laborales de los profesores que impartían clases en las tres escuelas analizadas. Además, se realizó un grupo focal con el personal que estuvo trabajando en el levantamiento de los datos partiendo de la siguiente pregunta de análisis: ¿Cuáles son las condiciones de trabajo de los maestros que laboran en escuelas primarias multigrado con rezago educativo?

\section{Resultados}

\section{Descripción de los docentes}

- Escuela general Ignacio Mori, de Pitahaya, Guaymas.

Profesor Pitahaya 1: La necesidad de un trabajo estable y fijo donde pudiera relacionarse con padres sin recursos económicos para útiles escolares y transporte fue lo que motivó el inicio de su labor docente. Al terminar su curso de educación indígena intensivo comenzó a dar clases en Belem o Pitahaya en la escuela multigrado, y después de un tiempo se cambió a Pótam donde trabajó en una escuela de organización comple- ta. Más tarde volvió a Pitahaya y continuó impartiendo clases en aulas multigrado. Además de estas comunidades, también ha laborado en Oroz, en Vícam, en Loma de Guamúchil y en Cárdenas, donde ha tenido la experiencia de trabajar en instituciones de organización completa y multigrado.

Mencionó que la administración o cambios de docentes entre las escuelas y el proceso para determinar directivos no se realizan con base en méritos o currículo académico del docente, sino por medio de lealtades objetivas hacia los altos mandos de la dirección de educación indígena. El maestro mantiene el incentivo de arraigo que él refiere como un estímulo para mantenerse dentro de la institución dando clases.

Profesora Pitahaya 2: Comenzó trabajando cuando estaba en la preparatoria en el Instituto Sonorense de Educación para Adultos (ISEA), organización que hizo un convenio con la Normal de Obregón, Sonora, para que les permitiera continuar la carrera y hacer el servicio social en el ISEA.

Terminó la licenciatura, pero sin titularse. Primero trabajó durante dos años en Huirivis; luego estuvo 4 años en el Baugo, cerca de las Guásimas; después de estas dos instituciones multigrado trabajó cuatro años en la costa de Hermosillo, en una escuela de organización completa, y de ahí pasó dos años en Bahía de Lobos antes de comenzar a dar clases en Pitahaya, pero debió suspender el servicio porque se movió de 
Vícam donde radica actualmente para cuidar a su madre, que estaba enferma en etapa terminal. Durante este periodo de tiempo la maestra fue reemplazada por la profesora Dionisia, quien estuvo impartiendo clases el segundo periodo de 2011 y el primer periodo de 2012 .

En cuanto a su experiencia personal, considera el trabajo de planeación en una escuela multigrado como lo más pesado porque debe adaptar los contenidos a diferentes grados al mismo tiempo; además, debido a que no existe un consenso sobre cuál es la mejor estrategia a seguir cada quien ajusta sus propias metodologías a las necesidades particulares de sus estudiantes y a su desempeño particular.

- Escuela Gabriel Zapajiza, de Huirivis, Guaymas

Profesora Huirivis 1: Empezó su labor profesional trabajando tres años en el magisterio de los cuales un año fue docente en Altar, Sonora, y dos años en Huirivis, uno de los cuales se desempeñó como directora. Ha participado en la planeación del colegiado entre los maestros de las comunidades aledañas como Pitahaya, Guásimas, Oroz, Huirivis, Rahum, actividad realizada por ellos mismos sin reconocimiento de la Secretaría y teniendo como fin lograr una planeación multigrado para compartir experiencias, métodos y material.

La maestra reporta que en la planeación didáctica debe considerar que existen temáticas que se extienden más de lo que se define porque en el mismo proceso surgen situaciones que requieren de diferentes aplicaciones y materiales, según las habilidades individuales entre los distintos grados a falta de competencias básicas que permita un equilibrio.

Profesora Huirivis 2: Comenzó trabajando como maestra en Vícam como interina; después trabajó en la Sierra de Álamos como promotora de educación inicial; ya con esa base, en el 2000 inicia su licenciatura en Educación en la Universidad Pedagógica Nacional (UPN); en el 2001 se traslada a Huirivis a educación inicial; un año más tarde por medio de una permuta pasa a Pitahaya con plaza de educación inicial. En el 2002 busca promoción para primaria y obtiene el cambio a Bahía de Lobos como directora; después de dos años la Secretaría de Educación y Cultura del Estado (SEC) le cambia la clave para docente de primaria y se dedica al grupo de tercer grado, además de ejercer como directora. Termina su licenciatura en el 2005 y continúa trabajando hasta el 2010 en Casa Blanca y pasa a Huirivis en el segundo ciclo de 2010.

Una de las principales razones por las cuales la maestra Pilar ha pedido sistemáticamente cambios de escuelas es buscando la cercanía con su hijo y la familia como red de apoyo para la crianza del niño. La maestra en su experiencia en el aula considera que es importante el manejo de las tecnologías de la información y comunicación (TIC). En su clase usa los recursos tecnológicos que tiene a su disposición, cuenta con cañón, PC además de Internet, esto le permite 
a los estudiantes recursos de búsqueda de información. Utiliza Enciclomedia como herramienta para complementar las temáticas para los distintos grados.

- Escuela José María Matus, de Rahum, Guaymas.

Profesora Rahum 1: Llegó a la escuela en el 2012 por medio de una permuta que realizó con el maestro que le precedió; menciona tener problemas con el sindicato porque no le han hecho cambio de educación inicial a clave de primaria, a pesar del tiempo que tiene de estar pidiéndolo.

Considera que el cambio de una escuela de organización completa a un multigrado sí es complicado porque ella piensa que en el primer caso se facilita la planeación porque solamente refiere a un grado. Mencionó que al planear sus clases en aulas multigrado enfoca las temáticas propias de los niños de tercer grado, que son los que están por salir al siguiente grupo, y que a partir de ahí va adecuando el nivel del contenido de la misma temática a los demás grados. A pesar de que la maestra tiene su domicilio en la comunidad de Rahum, menciona que estímulos económicos como el que se otorga a arraigo no les son entregados a maestros como ellos. Cuando regresó a la comunidad en donde reside y a la que pertenece, su pretensión era sentirse más segura y que sus hijos fueran educados en el seno de la familia extensa.

Profesor Rahum 2: El profesor tiene 12 años laborando en la escuela José María Matus. Cuan- do comenzó su trabajo, la escuela era unitaria e impartía clases a los seis grados de primaria, razón por la cual considera que fue un cambio muy difícil en la medida que tenía muchos años en Vícam y Batanconcita laborando en escuela de organización completa. Menciona que después de trabajar en la escuela multigrado con un grupo unitario, y basándose en el desempeño demostrado por sus estudiantes, la Secretaría de Educación le concedió un premio por sus méritos docentes.

Al hablar de la planeación de sus clases para los distintos grados que maneja en su salón, señala la importancia de manejar una sola temática para los tres grados conforme las necesidades particulares de cada grado ajustando los métodos de enseñanza o las actividades de clase y las tareas. El profesor dos es de los maestros que aún mantiene el incentivo de arraigo en su comunidad, lo cual le permite ofrecerles una atención más personalizada a los estudiantes, así como actividades extraclase.

Al impartir sus clases hace uso de las herramientas tecnológicas con las que cuenta en el aula, como PC, cañón y Smart Board; la escuela no cuenta con Internet, por lo que el maestro hace uso de Enciclomedia durante su jornada.

\section{Condiciones laborales}

El contenido de las entrevistas y observaciones realizadas se clasificaron mediante un análisis FODA donde se establecieron las fortalezas, oportunidades, debilidades y amenazas del con- 
texto en que laboran y las prácticas pedagógicas de los docentes de estas tres escuelas (ver en Anexos Tablas 6 y 7 ).

En laTabla 6 se observa que la amenaza para la educación multigrado en la zona yaqui está relacionada con la exclusión y olvido del municipio al cual pertenece en relación con la gestión de infraestructura y, por otro, lado la falta de atención de la Dirección de Educación Indígena. En relación con las demandas pedagógicas, particularmente las relacionadas con materiales educativos en la lengua y planeaciones específicas para grupos multigrado, las fortalezas se asocian a la unificación de la escuela-hogar por el acceso inmediato en las comunidades y la apropiación que los profesores hacen del currículo.

En la Tabla 7 se observan las condiciones de trabajo que indican como fortalezas: contar con una sola plaza, arraigo a la comunidad y el reducido número de alumnos. Las debilidades se enmarcan en la dificultad por exclusión y marginación geográfica de acceso a los programas de nivelación y capacitación para profesores. Estar en la periferia del municipio hace improbable la utilización de Unidades de Servicio de Apoyo a la Educación Regular (Usaer) para niños con capacidades deficientes.

\section{Discusión y conclusiones}

En síntesis, estas son las condiciones que describen las observaciones que se llevaron a cabo en las aulas de los profesores de las tres escuelas multigrado que en general enfatizan el trabajo docente siguiendo una dinámica integral, pero con una dependencia del alumno y una didáctica repetitiva centrada tanto en el tema de estudio como en el objeto de la clase en función de las diferencias en los niveles de la línea de entrada de las habilidades académicas de cada uno de los niños más que en la complejidad de los contenidos; los profesores mismos referían estar más interesados en las habilidades de los niños que en el nivel de complejidad asociado al grado escolar, y así se interesan en mejorar la comprensión de lectura o en su caso la escritura, atender los problemas de alfabetización que requiere el aula para lograr homogeneizar las competencias de lectoescritura, lo mismo que de matemáticas.

El sistema no es constructivista y es importante anotar que los profesores están muy centrados en el uso de herramientas repetitivas; en los ejercicios no utilizan material didáctico computarizado ni calculadora y se centran en el uso del pizarrón y la lengua yaqui para desarrollar su clase.

Fundamentalmente los materiales utilizados en pocas ocasiones son libros de la biblioteca de aula, de casa, periódicos murales y materiales que los mismos alumnos fueron diseñando a través del ciclo escolar sobre las temáticas desarrolladas por el maestro.

Las condiciones laborales del profesor como empleado son permanentes, goza de prestaciones y un salario, que además de ser constante, es importante en cantidad, y en el contexto de arrai- 
go a las comunidades tiene implicaciones para que su presencia sea percibida como autoridad y red de apoyo.

En lo didáctico, el apego de los alumnos tiene una relación vinculante con el uso permanente de la lengua nativa ya que promueve la identidad con la comunidad y los padres (Vera \& Montaño, 2003). Lo más relevante para los padres del arraigo y pertenencia del profesor a la comunidad yaqui, es, por supuesto, su papel y participación en las fiestas y rituales tradicionales. Sin embargo, la planeación, la falta de materiales, el excesivo trabajo de gestión directiva y el llenado de formatos en forma manual y electrónica son las debilidades que impactan el logro de los niños (Bertely \& Robles, 1997).

Un factor determinante en las condiciones que influyen en el buen desempeño del docente es la distribución del tiempo en las tareas como tal y como directivo dentro de la misma institución, ya que por los compromisos asociados a esta otra gestión debe viajar fuera de la comunidad a la supervisión de la zona, mientras los estudiantes a su cargo quedan sin clases o son asignados al otro salón lo que provoca un incorrecto seguimiento de su aprendizaje en los distintos grados.

En fin, la gestión directiva aumenta la carga en labor administrativa para el docente a tal punto que mencionan que los temas tratados en las reuniones de supervisión, no guardan relación con aspectos académicos o de planeación, sino, todo lo contrario: en estos espacios se le asigna más tarea administrativa lo que provoca su inasistencia a las reuniones.

Por tanto, la actividad como directivo debería ejercerla una persona asignada y evaluada para el cargo que lleve a cabo la función para tres escuelas multigrado de la misma zona. Este director debe tener como función vincular la institución académica con los demás organismos educativos, pero no solo para llevar una correcta burocracia empresarial, sino con el fin de incrementar los aprendizajes que puede ofrecer la escuela a sus estudiantes aun con las limitantes existentes en cuestión de infraestructura, material y capacitación.

El trabajo de observación y entrevista llevado a cabo nos permite descubrir primero: una dimensión semántica centrada en el profesor cuya identidad se revela a través de un análisis emics (Helfrich, 1995) sobre la manera como desarrolla su proceso de etnicidad y se integra a su historia de vida con la expectativa de vincularse a su comunidad (Dietz \& Mateos-Cortez, 2008). La otra dimensión es la pragmática, la cual es abordada como los estilos didácticos del profesor para promover procesos de aprendizaje; se desarrolla desde la perspectiva del habitus del docente ajustado a su circunstancia, con un cúmulo de competencias interculturales que le permiten promover y participar como igual en los procesos culturales de la comunidad con los alumnos (Bertely, 2000). Y finalmente presentamos una dimensión sincrética centrada en la 
relación entre maestros, alumnos, padres y comunidad, que articula el proceso de identidad y las prácticas sociales de interacción, y que en este caso se entrelazan y enriquecen permitiendo procesos de aprendizaje asociados a la cultura yaqui. Otros academicistas advierten que la institución gubernamental instituye como norma y desfasa la labor docente hacia procesos administrativos y evaluativos de regulación que desmejoran la atmósfera de convivencia y desvían la atención de lo trascendente hacia lo inmediato (Vera, Domínguez, Búrquez, Rodríguez \& Domínguez, 2004).

En conclusión, en la práctica, el maestro es un intermediario entre la cultura oficial y la propia; su trabajo se orienta hacia la enseñanza del español y la difusión de la cultura nacional. $\mathrm{Su}$ función se circunscribe a la transformación de la cultura indígena hacia la cultura y lengua nacional (Juárez, 2008).

Los docentes tienen que comprender lo que es cultura, vista como un elemento definitorio de los grupos humanos que transforman su entorno y sobreviven en él, y construyen su explicación del mundo y el sentido de su vida (Schmelkes, 2005).

El maestro requiere del entendimiento de la etnicidad como la conciencia de pertenecer a un pueblo que posee una cultura propia y distinta, que no es excluyente de otra, que se refiere al indigenismo, que es incluyente y permite un pro- ceso de identificación entre sí y de diferenciación con los "otros". En este contexto debe entenderse al maestro como facilitador de un proceso de reflexión: a través de la enseñanza de la segunda lengua, y no en el rol de emancipador y portador de un plan civilizador (Vera, 2006).

\section{Referencias}

Atkinson, P. \& Hammersley, M. (1994). Etnografia: métodos de investigación. Barcelona: Paidós Ibérica.

Bertely, M. (2000). El paradigma indigenista en crisis: voces étnicas, interculturalidad y educación en México. Mensajes universales de Las Américas para el siglo XXI. Conferencia presentada en 50 Congreso Internacional Americanista, ICA, Varsovia.

Bertely, M. \& Robles, A. (1997). Escolarización y etnicidad entre indigenas yalaltecos migrantes. Indígenas en la escuela. Conferencia llevada a cabo en el Consejo Mexicano de Investigación Educativa, Comie, México.

Búrquez, I., Domínguez, R. \& Vera, J. (2005). Interacción padres e hijos, la estimulación en el hogar y el desarrollo del niño en una zona rural de pobreza extrema. Trabajo presentado en el VIII Congreso Nacional de Investigación Educativa, Universidad de Sonora, del 30 de octubre al 02 de noviembre, México.

Comisión Nacional para el Desarrollo de los Pueblos Indígenas (2014). Programa Espe- 
cial de los Pueblos Indigenas 2014-2018. México: CDI. Recuperado desde www. cdi.gob.mx/index.php?option=com docman\&task=doc...Florescano, 1999

Dietz, G. \& Mateos-Cortez, L. (2008). El discurso intercultural ante el paradigma de la diversidad; estructuraciones subyacentes y migraciones discursivas del multiculturalismo contemporáneo. En S. Bastos (Ed.), Multiculturalismo y futuro en Guatemala (pp.23-54). Guatemala: Flacso y Oxfam.

Guerra, E. (2005). Los pueblos indígenas: entre la comunidad corporativa y el pluralismo 1968-2001. En B. Ilan \& L. Meyer (Eds.), Una historia contemporánea de México (pp.308-340). México: Océano.

Helfrich, H. (1995). Beyond the Dilemma of Cross-Cultural Psychology, Resolving the Tension between Etic and Emic Approaches. Culture \& Psychology, 5(2), 131153. DOI: $10.1177 / 1354067 X 9952002$.

Instituto Nacional de Estadística y Geografía (2010). Panorama sociodemográfico de México. México: Autor.

Instituto Nacional de Estadística y Geografía (2011). Marco conceptual del Censo de Población y Vivienda. México: Autor.

Instituto Nacional para la Evaluación de la Educación [INEE] (2006). Las primarias comunitarias. INEE Colección Cuadernos de Investigación, (26), 1-14.

Juárez, D. (2008). Educación en las pequeñas localidades rurales de México: logros y perspectivas. Gaceta Acontecer Educativo, 2, 3-17.
Juárez, D. (2009). Exclusión escolar en el medio rural. En R. González Návar (Comp.), Exclusión social y democracia. Los retos y las oportunidades. Guadalajara: Yoltéot.

Launtrey, J. (1985). Clase social, medio familiar e inteligencia (R. Royo, Trad.). Madrid: Aprendizaje Visor.

López, C. D. \& Viveros, R. (2002). Educación intercultural y pueblos indígenas en México. Elementos para analizar nuevas metáforas del siglo XXI. Agora Digital, (4), 15-21.

Peña, M. (2004). Crianza y desarrollo infantil en familias rurales de la región sur del Estado de Sonora. (Tesis de Maestría). Centro de Investigación en Alimentación y Desarrollo, A.C., Sonora.

Peña, M., Pérez, G. \& Vera, J. (2008). Desarrollo del niño en la zona indígena, su estimulación e interacción con sus padres. En AMEPSO (Eds.), La psicología social en México, 7, 735-740.

Robles, H., Hernández, J. \& Godínez, M. (2009). Estimaciones de la población indígena en México: conceptos y formas de cálculo. En COMIE (Eds.), Memoria Electrónica (CD-ROM) del X Congreso Nacional de Investigación Educativa (pp.1-10). Veracruz: Comie.

Rodríguez, C. (2006). Evaluación de la práctica docente en escuelas urbanas de educación primaria en Sonora. Tesis de maestría no publicada, en Desarrollo Regional. Centro de Investigación en Alimentación y Desarrollo, A.C., Sonora. 
Sánchez, O. (2011). La conciencia lingüística en estudiantes universitarios indigenas de inicios del siglo XXI. (Tesis de licenciatura). Ciudad de México: Universidad Nacional Autónoma de México.

Schmelkes, S. I. (1996). La evaluación de los centros sociales. Die, Civestav, ipn, MX. Recuperado desde http://ince.me.es/cumbre/dl.03.htm

Schmelkes, S. (2005). Educación intercultural. Informe de trabajo, UPN. Recuperado desde: http://interbilingue.ajusco.upn.mx/ modules.php? name $=$ News $\&$ file $=$ article\&si 12

Vera, J. A. (2006). Indigenismo y exclusión. Revista Ra Ximhai, 2(3), 677-681.

Vera, J. \& Domínguez, R. (2005). Práctica docente en el aula multigrado rural de una población mexicana. Educação e Pesquisa. Revista da Faculdade de Educação da USP, 31(1), 31-43.

Vera, J. A., Domínguez, R., Búrquez, K., Rodríguez, C. \& Domínguez, S. (2004). Un estudio comparativo de la práctica docente en la zona rural. Anuario de Investigaciones Educativas, 6, 35-50.

Vera, J. A. \& Montaño, A. (2003). Sociocultura y educación. En P. Sánchez, Aprendizaje y Desarrollo. La Investigación Educativa en México. México: Consejo Mexicano de Investigación Educativa.

Vera, J. A., Ramírez, R. \& Laborín, J. (2010). Diseño didáctico con enfoque colaborativo para escuelas de niños migrantes en Sonora. Estudios y Propuestas para el Medio Rural, 7, 139-158. 


\section{ANEXOS}

Tabla 1. Contexto socioeconómico y geográfico de las comunidades

\begin{tabular}{|l|c|c|c|}
\hline \multicolumn{1}{|c|}{ Nombre de la localidad al interior de cada municipio } & Rahum & Huirivis & Pitahaya \\
\hline Población total & 272 & 342 & 285 \\
\hline Viviendas particulares habitadas & 59 & 71 & 60 \\
\hline Porcentaje de población de 15 años o más, analfabeta & 17,22 & 15,31 & 23,46 \\
\hline Porcentaje de población de 15 años o más sin primaria completa & 52,57 & 42,0 & 54,9 \\
\hline Porcentaje de viviendas particulares sin escusado & 3,38 & 11,26 & 28,33 \\
\hline Porcentaje de viviendas particulares sin energía eléctrica & 3,38 & 10 & 15 \\
\hline Porcentaje de viviendas particulares sin agua entubada & 8,62 & 2,81 & 3,33 \\
\hline Promedio de ocupantes por cuarto en viviendas particulares habitadas & 1,56 & 1,77 & 1,93 \\
\hline Porcentaje de viviendas particulares con piso de tierra & 74,57 & 60,56 & 68,33 \\
\hline Porcentaje de viviendas particulares sin refrigerador & 54,23 & 49,29 & 71,66 \\
\hline
\end{tabular}

Fuente: Resultados de investigación

Tabla 2. Escuelas en observación en relación con el personal docente activo

\begin{tabular}{|c|c|c|c|c|c|}
\hline Escuela & Grado que atiende & Número de alumnos & Sexo & Director & Arraigo \\
\hline Escuela A & & & & & \\
\hline Profesor 1 & 1,2 y 3 & 23 & M & & X \\
\hline Profesor 2 & 4,5 y 6 & 19 & F & X & \\
\hline Escuela B & & & & & \\
\hline Profesor 1 & 1 y 2 & 21 & F & X & \\
\hline Profesor 2 & $3,4,5$ y 6 & 23 & & & \\
\hline Escuela C & & & F & & \\
\hline Profesor 1 & 1,2 y 3 & 21 & M & X & X \\
\hline Profesor 2 & 4,5 y 6 & 24 & & & \\
\hline
\end{tabular}

Fuente: Resultados de investigación

Tabla 3. Resultados de la prueba Enlace de la materia de Español

\begin{tabular}{|c|c|c|c|c|c|c|c|c|c|c|c|c|c|}
\hline \multirow[b]{2}{*}{ Grado } & \multirow[b]{2}{*}{ Año } & \multicolumn{3}{|c|}{ Insuficiente } & \multicolumn{3}{|c|}{ Elemental } & \multicolumn{3}{|c|}{ Bueno } & \multicolumn{3}{|c|}{ Excelente } \\
\hline & & Rah. & Hui. & Pit. & Rah. & Hui. & Pit. & Rah. & Hui. & Pit. & Rah. & Hui. & Pit. \\
\hline \multirow{3}{*}{ Tercero } & 2012 & $0 \%$ & $0 \%$ & 42,9 & 57,1 & $0 \%$ & 57,1 & 42,9 & 100 & $0 \%$ & $0 \%$ & $0 \%$ & $0 \%$ \\
\hline & 2011 & 88,9 & 80 & 28,6 & 11,1 & 20 & 14,3 & $0 \%$ & $0 \%$ & 14,3 & $0 \%$ & $0 \%$ & 42,9 \\
\hline & 2010 & 42,9 & 92,9 & 25 & 57,1 & 7,1 & 16,7 & $0 \%$ & $0 \%$ & 58,3 & $0 \%$ & $0 \%$ & $0 \%$ \\
\hline \multirow{3}{*}{ Sexto } & 2012 & 50 & 50 & 100 & 50 & 50 & $0 \%$ & $0 \%$ & $0 \%$ & $0 \%$ & $0 \%$ & $0 \%$ & $0 \%$ \\
\hline & 2011 & 42,9 & 75 & $0 \%$ & 42,9 & 25 & 28,6 & 14,3 & $0 \%$ & 57,1 & $0 \%$ & $0 \%$ & 14,3 \\
\hline & 2010 & $0 \%$ & $0 \%$ & $0 \%$ & 100 & 100 & 100 & $0 \%$ & $0 \%$ & $0 \%$ & $0 \%$ & $0 \%$ & $0 \%$ \\
\hline
\end{tabular}

Fuente: Resultados de investigación 
Tabla 4. Resultados de la prueba Enlace de la materia de Matemáticas

\begin{tabular}{|c|c|c|c|c|c|c|c|c|c|c|c|c|c|}
\hline \multirow[b]{2}{*}{ Grado } & \multirow[b]{2}{*}{ Año } & \multicolumn{3}{|c|}{ Insuficiente } & \multicolumn{3}{|c|}{ Elemental } & \multicolumn{3}{|c|}{ Bueno } & \multicolumn{3}{|c|}{ Excelente } \\
\hline & & Rah. & Hui. & Pit. & Rah. & Hui. & Pit. & Rah. & Hui. & Pit. & Rah. & Hui. & Pit. \\
\hline \multirow{3}{*}{ Tercero } & 2012 & 14,3 & $0 \%$ & 57,1 & 28,6 & $0 \%$ & 28,6 & 42,9 & 100 & 14,3 & 14,3 & $0 \%$ & $0 \%$ \\
\hline & 2011 & 55,6 & 80 & 28,6 & 44,4 & 20 & 14,3 & $0 \%$ & $0 \%$ & 28,6 & $0 \%$ & $0 \%$ & 28,6 \\
\hline & 2010 & 57,1 & 92,3 & 45,5 & 42,9 & 7,7 & 36,4 & $0 \%$ & $0 \%$ & 18,2 & $0 \%$ & $0 \%$ & $0 \%$ \\
\hline \multirow{3}{*}{ Sexto } & 2012 & 16,7 & 33,3 & 33,3 & 50 & 66,7 & 66,7 & 33,3 & $0 \%$ & $0 \%$ & $0 \%$ & $0 \%$ & $0 \%$ \\
\hline & 2011 & 25 & 75 & $0 \%$ & 62,5 & 25 & 25 & 12,5 & $0 \%$ & 85,7 & $0 \%$ & $0 \%$ & $0 \%$ \\
\hline & 2010 & $0 \%$ & $0 \%$ & 14,3 & 100 & 100 & 100 & $0 \%$ & $0 \%$ & $0 \%$ & $0 \%$ & $0 \%$ & $0 \%$ \\
\hline
\end{tabular}

Fuente: Resultados de investigación

Tabla 5. Guías de levantamiento de información en escuelas multigrado

\begin{tabular}{|l|c|c|c|}
\hline \multicolumn{1}{|c|}{ Guías } & Pitahaya (Ignacio Morí) & Huirivis (Gabriel Zapajiza) & Rahum (José Matus) \\
\hline Guía de entrevista para alumno & 13 & 17 & 15 \\
\hline Guía de entrevista para padres & 9 & 10 & 10 \\
\hline Guía de entrevista para maestro & 2 & 2 & 2 \\
\hline Guía de entrevista para director & 1 & 1 & 1 \\
\hline Guía de observación de infraestructura & 2 & 2 & 2 \\
\hline Guía de observación de actividades didácticas & 4 & 4 & 2 \\
\hline $\begin{array}{l}\text { Guía de organización y asistencia a apoyos } \\
\text { académicos extraclase }\end{array}$ & 2 & 2 & 2 \\
\hline
\end{tabular}

Fuente: Resultados de investigación

Tabla 6. Análisis FODA de las condiciones laborales y organizaciones de las escuelas

\begin{tabular}{|l|l|l|l|}
\hline \multicolumn{1}{|c|}{ Fortalezas } & \multicolumn{1}{|c|}{ Oportunidades } & \multicolumn{1}{c|}{ Debilidades } & \multicolumn{1}{c|}{ Amenazas } \\
\hline $\begin{array}{l}\text { Cooperativa operada por } \\
\text { maestras y/0 padres de fa- } \\
\text { milia }(\mathrm{A}, \mathrm{B}, \mathrm{C})\end{array}$ & $\begin{array}{l}\text { Libertad sobre la toma de decisiones de } \\
\text { currículos y planeación sin influencias de } \\
\text { sindicatos 0 supervisión }(\mathrm{A}, \mathrm{B}, \mathrm{C})\end{array}$ & $\begin{array}{l}\text { Perfil laboral, delimitación } \\
\text { temporal de actividades } \\
\text { como directivo y docente } \\
(\mathrm{A}, \mathrm{B}, \mathrm{C})\end{array}$ & $\begin{array}{l}\text { Existen lealtades objetivas hacia los altos mandos } \\
\text { de larección de educación indígena para el au- } \\
\text { sentismo, la planeación, la evaluación y el segui- } \\
\text { miento }(\mathrm{A}, \mathrm{B}, \mathrm{C})\end{array}$ \\
\hline & $\begin{array}{l}\text { Los niños puedes salir a comer a sus ca- } \\
\text { sas durante la hora de receso }(\mathrm{C})\end{array}$ & $\begin{array}{l}\text { Comunidades alejadas del centro de jurisdicción } \\
\text { del municipio de Guaymas }(\mathrm{A}, \mathrm{B}, \mathrm{C})\end{array}$ \\
\hline & & $\begin{array}{l}\text { La labor de supervisión es administrativa y no téc- } \\
\text { nica (A, B, C) }\end{array}$ \\
\hline & & $\begin{array}{l}\text { Falta de apoyo en gestión de recursos por la subse- } \\
\text { cretaría (A, B, C) }\end{array}$ \\
\hline
\end{tabular}

Nota: Escuela A (Gral. Ignacio Morí, Pitahaya); Escuela B (Gabriel Zapajiza, Huirivis); Escuela C (José Ma. Matus, Rahum)

Tabla 7. Análisis FODA de las condiciones de trabajo y pedagógicas de las escuelas

\begin{tabular}{|l|l|l|l|}
\hline \multicolumn{2}{|c|}{ Fortalezas } & \multicolumn{1}{|c|}{ Oportunidades } & \multicolumn{1}{c|}{ Debilidades } \\
\hline $\begin{array}{l}\text { Número de alumnos reducido }(\mathrm{A}, \\
\mathrm{B}, \mathrm{C})\end{array}$ & $\begin{array}{l}\text { Tener material didáctico }(\mathrm{A}, \\
\mathrm{B}, \mathrm{C})\end{array}$ & $\begin{array}{l}\text { No existen condiciones para trabajar con ni- } \\
\text { ños con problemas de aprendizaje }(\mathrm{A}, \mathrm{B}, \mathrm{C})\end{array}$ & $\begin{array}{l}\text { Capacitaciones en el manejo de tec- } \\
\text { nología didáctica }(\mathrm{A}, \mathrm{B}, \mathrm{C})\end{array}$ \\
\hline $\begin{array}{l}\text { Manejo de material didáctico en es- } \\
\text { pañol y lengua yaqui }(\mathrm{B}, \mathrm{C})\end{array}$ & $\begin{array}{l}\text { Maestros tienen una sola pla- } \\
\text { za. }(\mathrm{A}, \mathrm{B}, \mathrm{C})\end{array}$ & $\begin{array}{l}\text { Baja puntuación en ENLACE 2010-12. (B, } \\
\mathrm{C})\end{array}$ & $\begin{array}{l}\text { Falta de conocimientos sobre los } \\
\text { problemas de lectura }(\mathrm{A}, \text { B, C) }\end{array}$ \\
\hline $\begin{array}{l}\text { Permanencia y arraigo de los profe- } \\
\text { sores }(\mathrm{B}, \mathrm{C})\end{array}$ & $\begin{array}{l}\text { Los docentes voluntariamen- } \\
\text { te tienen programas de nive- } \\
\text { lación }(\mathrm{B}, \mathrm{C})\end{array}$ & Permanencia de los docentes $(\mathrm{A})$ & \\
\hline Experiencia docente $(\mathrm{B}, \mathrm{C})$ & & Docentes sin nivelación adecuada $(\mathrm{A})$ & \\
\hline
\end{tabular}

Nota: Escuela A (Gral. Ignacio Morí, Pitahaya), Escuela B (Gabriel Zapajiza, Huirivis); Escuela C (José Ma. Matus, Rahum) 